\title{
Model reference adaptive control of discrete-time piecewise linear systems
}

\author{
Mario di Bernardo ${ }^{2,3}$, Umberto Montanaro, ${ }^{1, *}$, Josep M. Olm ${ }^{4}$ and Stefania Santini ${ }^{2}$ \\ ${ }^{1}$ Istituto Motori, National Research Council, Naples 80125, Italy \\ ${ }^{2}$ Department of Systems and Computer Engineering, University of Naples Federico II, 80125 Naples, Italy \\ ${ }^{3}$ Department of Engineering Mathematics, University of Bristol, Bristol BS8 1TR, U.K. \\ ${ }^{4}$ Department of Applied Mathematics IV, Universitat Politècnica de Catalunya, 08800 Vilanova i la Geltrú, Spain
}

\begin{abstract}
SUMMARY
This article presents a switched MRAC controller for discrete-time piecewise linear systems. In the spirit of the work by Landau [1], the proof of asymptotic stability of the corresponding closed-loop error is carried out recasting its dynamics as a feedback system and showing the appropriate passivity features of the feedforward and the feedback paths. The challenge lies on the fact that both loops can be piecewise linear. Numerical results show excellent performance of the proposed controller even in the face of sudden variations of the plant parameters. Copyright (c) 2011 John Wiley \& Sons, Ltd.
\end{abstract}

Received ...

KEY WORDS: Discrete-time systems; model reference adaptive control; piecewise linear systems; passivity.

\section{INTRODUCTION}

Since the appearance of the pioneering work by Landau [1], Model Reference Adaptive Controllers (MRAC) have been used in a wide range of practical applications. MRAC is a powerful approach, yet simple to implement, which can guarantee robustness to parameter variations, noise and unmodelled dynamics. Given the increasing interest in the analysis and control of switched and hybrid systems [2], it is natural to seek an extension of the MRAC approach to Piecewise Affine (PWA) plants. Examples of MRAC schemes for PWA systems can be found in [3, 4, 5].

When considering MRAC schemes for Piecewise Smooth (PWS) systems, a major difficulty is to find appropriate tools to prove the asymptotic stability of the closed-loop error system which cannot be guaranteed using the classical approach because of the switched nature of the plant and, possibly, the control input and reference model. The recent extension of passivity theory to PWS systems presented in $[6,7]$ has made it possible to generalize to this class of systems proofs available for their smooth counterparts (see for example the proof in [8] and [5] for continuous-time PWA systems).

In this paper, we present a novel switched MRAC controller for discrete-time Piecewise Linear (PWL) systems. The controller extends to this class of systems the family of so-called Minimal Control Synthesis (MCS) algorithms [9] also available for smooth systems [10] and continuous-time PWA models [5]. The adaptive control law is based on three adaptive actions: a smooth feedforward term, a smooth feedback term and a switching adaptive feedback action. It can be used to make

\footnotetext{
${ }^{*}$ Correspondence to: Dr. Umberto Montanaro, Istituto Motori, National Research Council, Naples 80125, Italy. E-mail: u.montanaro@im.cnr.it 
a PWL plant track asymptotically the states of a smooth or PWL reference model. As shown in the paper, a proof of asymptotic stability can be obtained in both cases under the assumption that the reference model is quadratically stable (Q-stable) [11], that is, it admits a common quadratic Lyapunov function when unforced. Indeed, when this is verified, it is possible to follow the steps of Landaus' passivity-based classical proof and recast the closed-loop error system as a feedback system. The notable difference is that in our case, both the feedforward and feedback paths of such system can be PWA. Then, by using the notion of passivity for discrete-time Linear Time-Varying (LTV) systems in [12], we show that the MRAC scheme proposed in this paper renders the origin of the error system globally asymptotically stable.

The MRAC controller presented in the paper is validated on two representative examples: one where the reference model is Linear Time Invariant (LTI) while the plant is PWL and the other where both are PWL. In both cases, the controller guarantees excellent performance even in the presence of sudden variations of the plant parameters.

We wish to emphasize that other approaches are indeed possible to synthesize adaptive controllers for PWA systems. For example, in the case of matched nonlinearities it might be possible to use backstepping algorithms [13]. The control strategy presented in this paper is to address the existing gap in the theory of MRAC control approaches which makes their use unviable when the plant is not modelled by a smooth dynamical system. We believe extending the use of such controllers to nonsmooth systems is indeed theoretically relevant but also of practical use given the wide range of systems in applications affected by discontinuous events on a macroscopic timescale $[14,15,16]$.

The rest of the paper is outlined as follows. Section 2 contains the problem statement and introduces notation and a set of basic definitions. The main result of the article is in Section 3, where a switched adaptive strategy for MRAC control of discrete-time PWL plants is proposed. The proof of asymptotic stability of the corresponding closed-loop error dynamics is presented in Section 4. The assumption of Q-stability, which might be too strict for generic PWL reference models because it does not take into account this switching nature, is relaxed to Piecewise Quadratic stability (PWQstability) in Section 5. The numerical validation of the control algorithm is carried out in Section 6, while conclusions are drawn in Section 7. Finally, for the sake of completeness, basic results on passivity for discrete-time LTV systems are collected in Appendix A.

\section{PROBLEM STATEMENT AND DEFINITIONS}

Let the Euclidean space $\mathbb{R}^{n}$ be partitioned into $M$ domains $\left\{\Omega_{i}\right\}_{i \in \mathcal{M}}, \mathcal{M} \triangleq\{0,1, \ldots, M-1\}$, i.e. $\bigcup_{i=0}^{M-1} \Omega_{i}=\mathbb{R}^{n}$ and $\Omega_{i} \cap \Omega_{j}=\emptyset$ for all $i, j \in \mathcal{M}, i \neq j$.

We assume the plant to be described by a discrete time n-dimensional multi-modal PWL system of the form:

$$
x(k+1)=A_{i} x(k)+B u(k) \quad \text { if } \quad x(k) \in \Omega_{i}, \quad i \in \mathcal{M}
$$

where $x \in \mathbb{R}^{n}$ is the state vector, $u \in \mathbb{R}$ is the scalar input, and the matrices $A_{i}, B$, are assumed to be in the control canonical form given by

$$
A_{i}=\left[\begin{array}{cccc}
0 & 1 & \cdots & 0 \\
0 & 0 & \ddots & \vdots \\
\vdots & \vdots & & 1 \\
a_{i}^{(1)} & a_{i}^{(2)} & \cdots & a_{i}^{(n)}
\end{array}\right], \quad B=\left[\begin{array}{c}
0 \\
0 \\
\vdots \\
b
\end{array}\right], \quad \forall i \in \mathcal{M}
$$

with $b>0$. Apart from this hypothesis on the sign of $b$, all the entries on the last row of the plant matrices $A_{i}$ and $B$ may be completely unknown to the designer.

Let us also assume a multi-modal, PWL reference model for the plant of the form:

$$
\widehat{x}(k+1)=\widehat{A}_{\hat{i}} \widehat{x}(k)+\widehat{B} r(k) \quad \text { if } \quad \widehat{x}(k) \in \widehat{\Omega}_{\widehat{i}}
$$

where $\widehat{x} \in \mathbb{R}^{n}, \widehat{x}(0)=\widehat{x}_{0}, r \in \mathbb{R}$ is the input to the reference model, and $\left\{\widehat{\Omega}_{\widehat{i}}\right\}_{\widehat{i} \in \widehat{\mathcal{M}}}$, with $\widehat{\mathcal{M}} \triangleq$ $\{0,1, \ldots \widehat{M}-1\}$ being a partition of $\mathbb{R}^{n}$ defined in an analogous way as $\left\{\Omega_{i}\right\}_{i \in \mathcal{M}}$. As for the 
plant, the matrices of the reference model are chosen to be in the companion form given by:

$$
\widehat{A}_{\hat{i}}=\left[\begin{array}{cccc}
0 & 1 & \cdots & 0 \\
0 & 0 & \ddots & \vdots \\
\vdots & \vdots & & 1 \\
\widehat{a}_{\hat{i}}^{(1)} & \widehat{a}_{\hat{i}}^{(2)} & \cdots & \widehat{a}_{\hat{i}}^{(n)}
\end{array}\right], \quad \widehat{B}=\left[\begin{array}{c}
0 \\
0 \\
\vdots \\
\widehat{b}
\end{array}\right], \quad \forall \widehat{i} \in \widehat{\mathcal{M}} .
$$

We assume that the reference model (3) has a unique solution given an initial condition $\widehat{x}_{0}$.

It is also worth remarking that, whereas the partitions of both the plant and reference model phase spaces are assumed to be known, no specific structure on them is assumed. In fact, generically one may have that $M \neq \widehat{M}$, so the plant and reference model can be characterized by different numbers of phase space regions and associated vector fields.

The problem is to find an adaptive, state feedback control law $u(k)$ so as to ensure that the state variables of the plant, $x(k)$, track asymptotically the states, $\widehat{x}(k)$, of the reference model.

The remainder of the Section is devoted to introduce notation and basic definitions used hereafter. Let $\sigma_{i}(k), i \in \mathcal{M}$, and $\widehat{\sigma}_{i}(k), \widehat{i} \in \widehat{\mathcal{M}}$, be defined as

$$
\sigma_{i}(k)=\left\{\begin{array}{ll}
1 & \text { if } x(k) \in \Omega_{i}, \\
0 & \text { elsewhere }
\end{array} \quad \widehat{\sigma}_{\widehat{i}}(k)= \begin{cases}1 & \text { if } \widehat{x}(k) \in \widehat{\Omega}_{\widehat{i}} \\
0 & \text { elsewhere }\end{cases}\right.
$$

Note that, by the assumption of state measurements availability, the value of $\sigma_{i}(k)$ is known at every discrete-time instant, for every mode $i$.

\section{CONTROL STRATEGY}

The control problem posed in Section 2 can be solved by means of the switched adaptive strategy described in this Section.

Essentially, the control adaptation law presented in Theorem 1 below includes: (i) two smooth adaptive gains, that remain switched on whatever the modes in which the plant and reference model are evolving in (note that they have the same structure of the MCS gains proposed in [10] for discrete-time linear systems); (ii) a set of switching adaptive gains that take into account the PWS nature of the plant and the reference model: thus, they are actually switched on only when their trajectories enter certain phase-space domains.

Before stating the main result, let us establish the following assumption on the reference model.

\section{Assumption 1}

The reference model (3) is Q-stable [11], i.e. there exists a symmetric, positive definite matrix $P>0$ such that the following Linear Matrix Inequality (LMI) is verified:

$$
\widehat{A}_{\hat{i}}^{T} P \widehat{A}_{\widehat{i}}-P<0, \quad \forall \hat{i} \in \widehat{\mathcal{M}}
$$

\section{Theorem 1}

Given system (1) and a reference model of the form (3) such that Assumption 1 holds, the adaptive law:

$$
u(k)=L_{F B}(k) x(k)+L_{R}(k) r(k),
$$

where

$$
\begin{aligned}
L_{R}(k) & =\alpha \sum_{m=0}^{k} y_{e}(m+1) r(m)+\beta y_{e}(k+1) r(k), \\
L_{F B}(k) & =L_{0}(k)+L_{\Sigma}(k)+\widehat{L}_{\widehat{\Sigma}}(k),
\end{aligned}
$$


with

$$
\begin{aligned}
L_{0}(k) & =\alpha \sum_{m=0}^{k} y_{e}(m+1) x^{T}(m)+\beta y_{e}(k+1) x^{T}(k), \\
L_{\Sigma}(k) & =\sum_{i=1}^{M-1} \sigma_{i}(k) L_{i}(k), \quad \widehat{L}_{\widehat{\Sigma}}(k)=\sum_{\widehat{i}=1}^{\widehat{M}-1} \widehat{\sigma}_{\widehat{i}}(k) \widehat{L}_{\widehat{i}}(k), \\
L_{i}(k) & =\sum_{m=0}^{k} \chi_{i}(m), \quad \widehat{L}_{\widehat{i}}(k)=\sum_{m=0}^{k} \widehat{\chi}_{\widehat{i}}(m),
\end{aligned}
$$

and

$$
\begin{aligned}
& \chi_{i}(m)=\left\{\begin{array}{l}
\rho y_{e}(m+1) x^{T}(m), \text { if } \quad x(m) \in \Omega_{i}, \\
0, \quad \text { elsewhere }
\end{array}\right. \\
& \widehat{\chi}_{\hat{i}}(m)=\left\{\begin{array}{l}
\rho y_{e}(m+1) x^{T}(m), \\
0, \quad \text { elsewhere }
\end{array}\right.
\end{aligned}
$$

with $\alpha, \beta, \rho>0$ and

$$
y_{e} \triangleq B_{e}^{T} P x_{e}, \quad x_{e} \triangleq \widehat{x}-x, \quad B_{e}=(0, \ldots, 0,1)^{T} \in \mathbb{R}^{n},
$$

guarantees the global asymptotic stability of the closed-loop error dynamics.

\section{Remark 1}

Note that Assumption 1 is not as restrictive as it might appear at first, since the reference model is typically selected by design. Indeed, in many practical cases the aim of the control action is to compensate the PWS nature of the plant. Hence, an LTI system is usually selected as reference model and, for such a case, Assumption 1 simply reduces to demand that the reference model matrix $\widehat{A}$ be Hurwitz. Moreover, for LTI reference models, the proposed control law improves that of [10], because the former does not require slow variation of the plant parameters with respect to the adaptive gains.

\section{PROOF OF THEOREM 1}

In what follows, Theorem 1 is proved using passivity theory. For the sake of completeness, some fundamental results about discrete-time LTV passive systems are collected in Appendix A.

The main idea is to show that the closed-loop system can be recast as a feedback loop of two passive switched systems without input. In so doing, the closed-loop error system is passive and therefore stable. Furthermore, exploiting the passivity properties of the feedforward and feedback blocks, we prove the asymptotic convergence of the tracking error dynamics towards the origin from any initial condition.

The proof is based on the following four steps:

1. Recast the error dynamics as a feedback system.

2. Prove strict passivity of the feedforward dynamics.

3. Prove passivity of the feedback block.

4. Prove asymptotic convergence of the closed-loop error system.

The next subsections are devoted to each one of the above indicated steps. Assumption 1 is supposed to be fulfilled throughout the proof. 


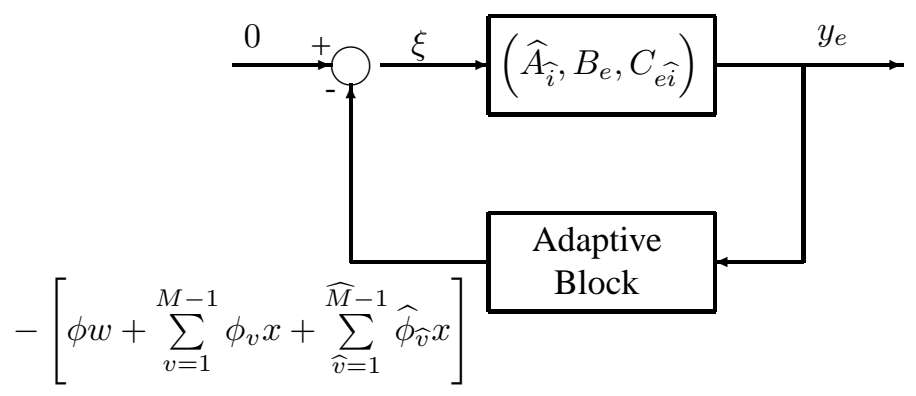

Figure 1. Schematization of the closed loop error dynamics

\subsection{Step 1: Recast the error dynamics as a feedback system.}

Given the plant and the reference dynamics (1) and (3), respectively, using the definition of the control strategy in (7) and the expression of the tracking error in (15), we obtain

$$
x_{e}(k+1)=\widehat{A}(k) x_{e}(k)+B_{e}\left[\phi(k) w(k)+\sum_{v=1}^{M-1} \phi_{v}(k) x(k)+\sum_{\widehat{v}=1}^{\widehat{M}-1} \widehat{\phi}_{\widehat{v}}(k) x(k)\right],
$$

where

$$
\widehat{A}(k)=\widehat{A}(\widehat{x}(k)):=\widehat{A}_{\widehat{i}} \quad \text { when } \widehat{x}(k) \in \widehat{\Omega}_{\widehat{i}},
$$

and

$$
\begin{gathered}
\phi(k)=\left[B_{e}^{T}\left(\widehat{A}_{0}-A_{0}\right)-b L_{0}(k) \vdots \widehat{b}-b L_{R}(k)\right], \quad w(k) \triangleq\left[x^{T}(k) r(k)\right]^{T}, \\
\phi_{v}(k) \triangleq \sigma_{v}(k)\left[B_{e}^{T} \Delta A_{v}-b L_{v}(k)\right], \quad \Delta A_{v} \triangleq A_{0}-A_{v}, \quad v=1,2, \ldots, M-1, \\
\widehat{\phi}_{\widehat{v}}(k) \triangleq \widehat{\sigma}_{\widehat{v}}(k)\left[B_{e}^{T} \Delta \widehat{A}_{\widehat{v}}-b \widehat{L}_{\widehat{v}}(k)\right], \quad \Delta \widehat{A}_{\widehat{v}} \triangleq \widehat{A}_{\widehat{v}}-\widehat{A}_{0}, \quad \widehat{v}=1,2, \ldots \widehat{M}-1 .
\end{gathered}
$$

It is worth emphasizing that the key point that yields (16) is that the matrices $A_{i}, \widehat{A}_{\bar{i}}$ are assumed to be in control canonical form (recall (2) and (4), respectively), which entails that $\widehat{A}_{0}-A_{0}, \Delta A_{v}$ and $\Delta \widehat{A}_{\widehat{v}}$ are matrices with null elements everywhere but in the last row. Further mathematical details can be found in Appendix B.

From (16), the error dynamics can be easily represented as the feedback system shown in Figure 1. Here the feedforward block is described by:

$$
\Sigma_{0}:=\left\{\begin{array}{cl}
x_{e}(k+1) & =\widehat{A}(k) x_{e}(k)+B_{e} \xi(k+1) \\
y_{e}(k) & =B_{e}^{T} P x_{e}(k),
\end{array}\right.
$$

while the feedback block has input $y_{e}(k)$ and output $-\xi(k)$, with

$$
\xi(k+1)=\phi(k) w(k)+\sum_{v=1}^{M-1} \phi_{v}(k) x(k)+\sum_{\widehat{v}=1}^{\widehat{M}-1} \widehat{\phi}_{\widehat{v}}(k) x(k) .
$$

\subsection{Step 2: Passivity of the feedforward dynamics.}

We want to prove passivity of the feedforward block $\Sigma_{0}$ given by (21)-(17). To this aim, let us consider the following systems:

$$
\begin{aligned}
& \Sigma_{1}:=\left\{\begin{array}{ccc}
\tilde{x}_{e}(k+1) & =\widehat{A}(k) \tilde{x}_{e}(k)+\widehat{A}(k) B_{e} \xi(k), \\
y_{e 1}(k) & =B_{e}^{T} P \tilde{x}_{e}(k)+\frac{1}{2} B_{e}^{T} P B_{e} \xi(k),
\end{array}\right. \\
& \Sigma_{2}:=y_{e 2}(k)=\frac{1}{2} B_{e}^{T} P B_{e} \xi(k) .
\end{aligned}
$$




\section{Lemma 1}

The parallel connection of systems $\Sigma_{1}$ and $\Sigma_{2}$ is input-output equivalent to system $\Sigma_{0}$.

Proof

The change of variables

$$
x_{e}(k)=\tilde{x}_{e}(k)+B_{e} \xi(k)
$$

in $\Sigma_{0}(21)$ yields

$$
\begin{array}{cl}
\tilde{x}_{e}(k+1) & =\widehat{A}(k) \tilde{x}_{e}(k)+\widehat{A}(k) B_{e} \xi(k), \\
y_{e}(k) & =B_{e}^{T} P \tilde{x}_{e}(k)+B_{e}^{T} P B_{e} \xi(k) .
\end{array}
$$

Then, it is straightforward to show that $y_{e 1}(k)+y_{e 2}(k)=y_{e}(k)$.

Lemma 2

The parallel connection of systems $\Sigma_{1}$ and $\Sigma_{2}$ is input strictly passive, with common quadratic storage function

$$
V(\tilde{x}(k))=\frac{1}{2} \tilde{x}^{T}(k) P \tilde{x}(k)
$$

\section{Proof}

We can prove passivity of $\Sigma_{1}$ using Lemma 3.i in Appendix A. Notice that Assumption 1 yields

$$
\widehat{A}^{T}(k) P \widehat{A}(k)-P=-Q(k),
$$

with $Q(k)$ positive definite, which means that (79) is fulfilled. Indeed, (17) allows to define

$$
Q(k)=Q_{\widehat{i}}=P-\widehat{A}_{\widehat{i}}^{T} P \widehat{A}_{\widehat{i}} \quad \text { when } \widehat{x}(k) \in \widehat{\Omega}_{\widehat{i}},
$$

with $Q(k)$ being trivially symmetric. Moreover, (80) and (81) are also verified with:

$$
\begin{aligned}
S^{T}(k) & =B_{e}^{T} P-\left(\widehat{A}(k) B_{e}\right)^{T} P \widehat{A}(k)=B_{e}^{T} Q(k), \\
R(k) & =\frac{1}{2} B_{e}^{T} P B_{e}+\left(\frac{1}{2} B_{e}^{T} P B_{e}\right)^{T}-\left(\widehat{A}(k) B_{e}\right)^{T} P \widehat{A}(k) B_{e}=B_{e}^{T} Q(k) B_{e} .
\end{aligned}
$$

Then matrix $M(k)$ in (82) can be obtained as:

$$
M(k)=\left(\begin{array}{cc}
Q(k) & Q^{T}(k) B_{e} \\
B_{e}^{T} Q(k) & B_{e}^{T} Q(k) B_{e}
\end{array}\right)=\left(\begin{array}{cc}
Q(k) & Q(k) B_{e} \\
B_{e}^{T} Q(k) & B_{e}^{T} Q(k) B_{e}
\end{array}\right), \quad \forall k \geq 0 .
$$

Finally, the positive semidefinite character of $M(k), \forall k \geq 0$, is shown as follows:

$$
\begin{aligned}
{\left[\tilde{x}_{e}^{T}(k) \xi(k)\right] M(k)\left[\begin{array}{c}
\tilde{x}_{e}(k) \\
\xi(k)
\end{array}\right] } & =\left[\tilde{x}_{e}(k)+B_{e} \xi(k)\right]^{T} Q(k)\left[\tilde{x}_{e}(k)+B_{e} \xi(k)\right] \\
& =x_{e}^{T}(k) Q(k) x_{e}(k) \geq 0, \quad \forall \tilde{x}_{e}(k), \xi(k) .
\end{aligned}
$$

Hence, Lemma 3.i in Appendix A yields passivity of $\Sigma_{1}$ and, consequently, Lemma 3.ii entails:

$$
y_{e 1}(k) \xi(k)=V\left(\tilde{x}_{e}(k+1)\right)-V\left(\tilde{x}_{e}(k)\right)+\frac{1}{2} x_{e}^{T}(k) Q(k) x_{e}(k)
$$

Now, for the parallel connection of $\Sigma_{1}$ and $\Sigma_{2}$, from (24) and (29) we have:

$$
\begin{aligned}
y_{e}(k) \xi(k)= & y_{e 1}(k) \xi(k)+y_{e 2}(k) \xi(k)=V\left(\tilde{x}_{e}(k+1)\right)-V\left(\tilde{x}_{e}(k)\right)+ \\
& \frac{1}{2} x_{e}^{T}(k) Q(k) x_{e}(k)+\frac{1}{2} \xi^{T}(k) B_{e}^{T} P B_{e} \xi(k) .
\end{aligned}
$$

Then,

$$
y_{e}(k) \xi(k)=V\left(\tilde{x}_{e}(k+1)\right)-V\left(\tilde{x}_{e}(k)\right)+\frac{1}{2} x_{e}^{T}(k) Q(k) x_{e}(k)+\frac{1}{2} p_{n n} \xi^{2}(k),
$$

where $p_{n n}$ denotes the matrix element of $P$ located in the $(n, n)$ position. Notice that $p_{n n}>0$ because $P$ is positive definite and, as $Q(k)$ is also positive definite for all $k \geq 0$, the result follows. 


\subsection{Step 3: Passivity of the feedback dynamics.}

Let us first introduce notation and basic definitions used in the proof.

For the switching instants of both the plant and the reference model we use the standard notation adopted in [17]. More precisely, the activation sequence of the plant is given by:

$$
\Sigma=\left\{x_{0},\left(q_{0}, k_{0}\right),\left(q_{1}, k_{1}\right), \ldots\left(q_{p}, k_{p}\right) \ldots \quad \mid \quad q_{p} \in \mathcal{M}, p \in \mathbb{N}\right\},
$$

in which $k_{0}=0$ is the initial discrete time and $x_{0}$ is the initial state. Then, when $k \in\left[k_{p} ; k_{p+1}\right)$ it is $x(k) \in \Omega_{q_{p}}$ by definition, that is, the $q_{p}$-th subsystem is active. The sequence $\Sigma$ may or may not be infinite; in the finite case we may take $k_{p+1}=\infty$.

For any $i \in \mathcal{M}$ we denote

$$
\Sigma / i=\left\{k_{i_{1}}, k_{i_{2}}, \ldots k_{i_{s}}, \ldots \mid q_{i_{s}}=i, s \in \mathbb{N}\right\},
$$

the sequence of switching times when the $i$-th subsystem is activated, and thus

$$
\left\{k_{i_{1}+1}-1, k_{i_{2}+1}-1, \ldots k_{i_{s}+1}-1, \ldots \quad \mid \quad q_{i_{s}}=i, s \in \mathbb{N}\right\}
$$

is the sequence of the discrete time instants when the $i$-th subsystem was last active. Analogously, we define

$$
\widehat{\Sigma}=\left\{\widehat{x}_{0},\left(\widehat{q}_{0}, \widehat{k}_{0}\right),\left(\widehat{q}_{1}, \widehat{k}_{1}\right), \ldots\left(\widehat{q}_{p}, \widehat{k}_{p}\right) \ldots \mid \widehat{q}_{p} \in \widehat{M}, p \in \mathbb{N}\right\}
$$

as the activation sequence of the reference model with $\widehat{k}_{0}=0$. Moreover, for any $\widehat{i} \in \widehat{\mathcal{M}}$,

$$
\widehat{\Sigma} \widehat{i}=\left\{\widehat{k}_{\widehat{i}_{1}}, \widehat{k}_{\hat{i}_{2}}, \ldots \widehat{k}_{\hat{i}_{s}}, \ldots \quad \mid \quad \widehat{q}_{\hat{i}_{s}}=\widehat{i}, s \in \mathbb{N}\right\}
$$

is defined as the sequence of switching times when the $\widehat{i}$-th subsystem of the reference model is activated, and analogously,

$$
\left\{\widehat{k}_{\widehat{i}_{1}+1}-1, \widehat{k}_{\widehat{i}_{2}+1}-1, \ldots \widehat{k}_{\widehat{i}_{s}+1}-1, \ldots \mid \widehat{q}_{i_{s}}=\widehat{i}, s \in \mathbb{N}\right\}
$$

is the sequence of time instants when the $\widehat{i}$-th subsystem was last active.

Finally, given a generic vector $\eta=\left[\begin{array}{llll}\eta_{1} & \eta_{2} & \cdots & \eta_{m}\end{array}\right]^{T} \in \mathbb{R}^{m}$ we denote as $\tilde{\Gamma}(\eta)$ the $m$ dimensional vector defined as:

$$
\tilde{\Gamma}(\eta)=\left[\begin{array}{lllll}
0 & \eta_{1} & \eta_{2} & \cdots & \eta_{m-1}
\end{array}\right]^{T} .
$$

Passivity of the feedback block depicted in Figure 1 will be proved showing that Popov inequality [1] is fulfilled. Namely, that for some finite constant $\gamma$,

$$
\sum_{k=0}^{l} y_{e}(k+1)[-\xi(k+1)] \geq-\gamma^{2}, \quad \forall l \geq 0,
$$

i.e. that

$$
\sum_{k=0}^{l}-y_{e}(k+1)\left[\phi(k) w(k)+\sum_{v=1}^{M-1} \phi_{v}(k) x(k)+\sum_{\widehat{v}=1}^{\widehat{M}-1} \widehat{\phi}_{\widehat{v}}(k) x(k)\right] \geq-\gamma^{2} .
$$

The previous inequality can be recast as:

$$
S_{0}+S+\widehat{S} \geq-\gamma^{2}
$$


where

$$
\begin{aligned}
S_{0} & =\sum_{k=0}^{l}-y_{e}(k+1) \phi(k) w(k) \\
S & =\sum_{v=1}^{M-1} S_{v}, \quad S_{v}=\sum_{k=0}^{l}-y_{e}(k+1) \phi_{v}(k) x(k), \\
\widehat{S} & =\sum_{\widehat{M}=1}^{\widehat{M}_{\widehat{v}}-1} \widehat{S}_{\widehat{v}}, \quad \widehat{S}_{\widehat{v}}=\sum_{k=0}^{l}-y_{e}(k+1) \widehat{\phi}_{\widehat{v}}(k) x(k)
\end{aligned}
$$

Now, since $\phi(k)$ does not switch, by choosing the adaptive gains $L_{R}$ and $L_{0}$ as in (8) and (10), from the proof of the discrete time MCS in [10], it follows that:

$$
\sum_{k=0}^{l}-y_{e}(k+1) \phi(k) w(k) \geqslant-\gamma_{1}^{2}
$$

with $\gamma_{1}$ being some real finite constant. Notice also that, indeed, as $\phi(k)$ does not depend on $A(k)$ but on $A_{0}$ (see (18)), the slow variation of the plant parameters with respect to the adaptive gains assumed in [10] to prove (43) is not required here, as claimed in Remark 1.

It is then evident that, if we prove that $S_{v} \geq-\gamma_{v}^{2}$ in (41) with $v=1,2 \ldots M-1$ and $\widehat{S}_{\widehat{v}} \geq-\widehat{\gamma}_{\widehat{v}}^{2}$ in (42) with $\widehat{v}=1,2 \ldots \widehat{M}-1$, where $\gamma_{v}$ and $\widehat{\gamma}_{\widehat{v}}$ are some scalar constants, then (38) is verified and consequently the proof of the theorem is completed.

Now we consider the term $S$ in (41) and focus onto a generic addend $S_{v}$ which can be written as:

$$
S_{v}=\sum_{j=1}^{n} S_{v}^{(j)}, \quad S_{v}^{(j)} \triangleq \sum_{k=0}^{l}-y_{e}(k+1) \phi_{v}^{(j)}(k) x^{(j)}(k)
$$

with $j=1,2 \ldots n, v=1, \ldots M-1$ and

$$
\phi_{v}^{(j)}(k)=\sigma_{v}(k)\left[\delta_{v}^{(j)}-b L_{v}^{(j)}(k)\right], \quad \delta_{v}^{(j)} \triangleq a_{0}^{(j)}-a_{v}^{(j)} .
$$

Considering (5) and the notation adopted to describe the switching instants used throughout the paper, the term $S_{v}^{(j)}$ has the following form:

$$
S_{v}^{(j)}=\left\{\begin{array}{l}
\sum_{s=1}^{\theta_{v}} \sum_{k=k_{v_{s}}}^{k_{v_{s}+1}-1}-y_{e}(k+1)\left[\delta_{v}^{(j)}-b L_{v}^{(j)}(k)\right] x^{(j)}(k), \text { if } x(l) \notin \Omega_{v}, \\
\sum_{s=1}^{\theta_{v}} \sum_{k=k_{v_{s}}}^{k_{v_{s}+1}-1}-y_{e}(k+1)\left[\delta_{v}^{(j)}-b L_{v}^{(j)}(k)\right] x^{(j)}(k)+ \\
+\sum_{k=k_{v}\left(\theta_{v}+1\right)}^{l}-y_{e}(k+1)\left[\delta_{v}^{(j)}-b L_{v}^{(j)}(k)\right] x^{(j)}(k) \text { if } x(l) \in \Omega_{v},
\end{array}\right.
$$

where $\theta_{v}$ is the generic number of times the trajectory left $\Omega_{v}$.

Let us now focus on the most complex case $x(l) \in \Omega_{v}$ : from (12) and (13) we have

$$
\begin{aligned}
& L_{v}^{(j)}(k)-L_{v}^{(j)}(k-1)=\rho y_{e}(k+1) x^{(j)}(k) \Rightarrow \\
& \Rightarrow-y_{e}(k+1) x^{(j)}(k)=\frac{1}{\rho b}\left[b L_{v}^{(j)}(k-1)-b L_{v}^{(j)}(k)\right] .
\end{aligned}
$$


The substitution of (47) in (46) for $x(l) \in \Omega_{v}$ yields:

$$
\begin{aligned}
S_{v}^{(j)} & =\frac{1}{\rho b} \sum_{s=1}^{\theta_{v}} \sum_{k=k_{v_{s}}}^{k_{v_{s}+1}-1}\left[b L_{v}^{(j)}(k-1)-b L_{v}^{(j)}(k)\right]\left[\delta_{v}^{(j)}-b L_{v}^{(j)}(k)\right] \\
& +\frac{1}{\rho b} \sum_{k=k_{v}\left(\theta_{v}+1\right)}^{l}\left[b L_{v}^{(j)}(k-1)-b L_{v}^{(j)}(k)\right]\left[\delta_{v}^{(j)}-b L_{v}^{(j)}(k)\right] .
\end{aligned}
$$

Defining now

$$
\psi_{v}^{(j)}(k)=\delta_{v}^{(j)}-b L_{v}^{(j)}(k), \quad v=1,2, \ldots M-1, / j=1,2, \ldots n,
$$

the generic term $S_{v}^{(j)}$ in (48) can be expressed as:

$$
\begin{aligned}
S_{v}^{(j)}= & \frac{1}{\rho b} \sum_{s=1}^{\theta_{v}} \sum_{k=k_{v_{s}}}^{k_{v_{s}+1}-1}\left[\psi_{v}^{(j)}(k)-\psi_{v}^{(j)}(k-1)\right] \psi_{v}^{(j)}(k)+ \\
& +\frac{1}{\rho b} \sum_{k=k_{v_{\left(\theta_{v}+1\right)}}}^{l}\left[\psi_{v}^{(j)}(k)-\psi_{v}^{(j)}(k-1)\right] \psi_{v}^{(j)}(k) .
\end{aligned}
$$

Taking into account the definition in (12), it is evident that when the state of the plant does not belong to $\Omega_{i}$, with $i \in \mathcal{M} \backslash\{0\}$, the gain $L_{i}$ is not varied. Similarly, when the reference state does not belong to $\widehat{\Omega}_{\widehat{i}}$, with $\widehat{i} \in \widehat{\mathcal{M}} \backslash\{0\}$, the gain $\widehat{L}_{\widehat{i}}$ is kept constant. Hence, in accordance with the notation used to describe the switching instants, we have:

$$
\begin{aligned}
L_{i}\left(k_{i_{s}}-1\right) & =L_{i}\left(k_{i_{s-1}+1}-1\right), \quad s \in \mathbb{N} \backslash\{1\}, \\
\widehat{L}_{\widehat{i}}\left(k_{\hat{i}_{s}}-1\right) & =\widehat{L}_{\widehat{i}}\left(k_{\hat{i}_{s-1}+1}-1\right), \quad s \in \mathbb{N} \backslash\{1\} .
\end{aligned}
$$

Therefore, notice that, from (52), we have:

$$
\psi_{v}^{(j)}\left(k_{v_{s}}-1\right)=\psi_{v}^{(j)}\left(k_{v_{s-1}+1}-1\right), \quad s=2,3, \ldots \theta_{v}+1 .
$$

Collecting all the samples in the vector $\Psi_{v}^{(j)}$, defined as

$$
\begin{array}{cccccc}
\Psi_{v}^{(j)} \triangleq & {\left[\begin{array}{ccccc}
\psi_{v}^{(j)}\left(k_{v_{1}}\right) & \psi_{v}^{(j)}\left(k_{v_{1}}+1\right) & \cdots & \psi_{v}^{(j)}\left(k_{v_{1}+1}-1\right) & \vdots \\
\psi_{v}^{(j)}\left(k_{v_{2}}\right) & \psi_{v}^{(j)}\left(k_{v_{2}}+1\right) & \cdots & \psi_{v}^{(j)}\left(k_{v_{2}+1}-1\right) & \vdots \\
\cdots & \cdots & \cdots & \cdots & \cdots \\
\psi_{v}^{(j)}\left(k_{v_{\theta_{v}}}\right) & \psi_{v}^{(j)}\left(k_{v_{\theta_{v}}}+1\right) & \cdots & \psi_{v}^{(j)}\left(k_{v_{\theta_{v}}+1}-1\right) & \vdots \\
\psi_{v}^{(j)}\left(k_{v_{\left(\theta_{v}+1\right)}}\right) & \psi_{v}^{(j)}\left(k_{v_{\left(\theta_{v}+1\right)}}+1\right) & \cdots & \psi_{v}^{(j)}(l-1) & \psi_{v}^{(j)}(l)
\end{array}\right]^{T},}
\end{array}
$$

and taking into account definition (37), we have:

$$
\begin{gathered}
\tilde{\Gamma}\left(\Psi_{v}^{(j)}\right)= \\
\left.\begin{array}{ccccccc}
0 & \psi_{v}^{(j)}\left(k_{v_{1}}\right) & \psi_{v}^{(j)}\left(k_{v_{1}}+1\right) & \cdots & \psi_{v}^{(j)}\left(k_{v_{1}+1}-2\right) & \vdots \\
\psi_{v}^{(j)}\left(k_{v_{1}+1}-1\right) & \psi_{v}^{(j)}\left(k_{v_{2}}\right) & \cdots & \psi_{v}^{(j)}\left(k_{v_{2}+1}-2\right) & \vdots \\
\cdots & \cdots & \cdots & \cdots & \cdots \\
& \psi_{v}^{(j)}\left(k_{v_{\left(\theta_{v}-1\right)}+1}-1\right) & \psi_{v}^{(j)}\left(k_{v_{\theta_{v}}}\right) & \cdots & \psi_{v}^{(j)}\left(k_{v_{v}+1}-2\right) & \vdots \\
& \psi_{v}^{(j)}\left(k_{v_{\theta_{v}}+1}-1\right) & \psi_{v}^{(j)}\left(k_{v_{\left(\theta_{v}+1\right)}}\right) & \cdots & \psi_{v}^{(j)}(l-2) & \psi_{v}^{(j)}(l-1)
\end{array}\right]^{T} .
\end{gathered}
$$


From (54), expression (56) can be written as:

$$
\begin{gathered}
\tilde{\Gamma}\left(\Psi_{v}^{(j)}\right)= \\
\begin{array}{ccccccc}
0 & \psi_{v}^{(j)}\left(k_{v_{1}}\right) & \psi_{v}^{(j)}\left(k_{v_{1}}+1\right) & \cdots & \psi_{v}^{(j)}\left(k_{v_{1}+1}-2\right) & \vdots \\
\psi_{v}^{(j)}\left(k_{v_{2}}-1\right) & \psi_{v}^{(j)}\left(k_{v_{2}}\right) & \cdots & \psi_{v}^{(j)}\left(k_{v_{2}+1}-2\right) & \vdots \\
\cdots & \cdots & \cdots & \cdots & \cdots
\end{array} \\
\left.\begin{array}{cccccc}
\psi_{v}^{(j)}\left(k_{v_{\theta_{v}}}-1\right) & \psi_{v}^{(j)}\left(k_{v_{\theta_{v}}}\right) & \cdots & \psi_{v}^{(j)}\left(k_{v_{\theta_{v}}+1}-2\right) & \vdots \\
\psi_{v}^{(j)}\left(k_{v_{\left(\theta_{v}+1\right)}}-1\right) & \psi_{v}^{(j)}\left(k_{v_{\left(\theta_{v}+1\right)}}\right) & \cdots & \psi_{v}^{(j)}(l-2) & \psi_{v}^{(j)}(l-1)
\end{array}\right]^{T} .
\end{gathered}
$$

Now, from (55) and (57), the generic addend of $S_{v}$ can be rewritten as:

$$
S_{v}^{(j)}=\frac{1}{\rho b}\left[\left\|\Psi_{v}^{(j)}\right\|^{2}-\tilde{\Gamma}^{T}\left(\Psi_{v}^{(j)}\right) \Psi_{v}^{(j)}-\psi_{v}^{(j)}\left(k_{v_{1}}-1\right) \psi_{v}^{(j)}\left(k_{v_{1}}\right)\right] .
$$

Considering that $\|\tilde{\Gamma}(\eta)\| \leqslant\|\eta\|$ :

$$
\begin{aligned}
& \tilde{\Gamma}^{T}\left(\Psi_{v}^{(j)}\right) \Psi_{v}^{(j)} \leq\left|\tilde{\Gamma}^{T}\left(\Psi_{v}^{(j)}\right) \Psi_{v}^{(j)}\right| \leq\left\|\tilde{\Gamma}^{T}\left(\Psi_{v}^{(j)}\right)\right\|\left\|\Psi_{v}^{(j)}\right\| \leq\left\|\Psi_{v}^{(j)}\right\|^{2} \Rightarrow \\
& \Rightarrow \quad-\tilde{\Gamma}^{T}\left(\Psi_{v}^{(j)}\right) \Psi_{v}^{(j)} \geq-\left\|\Psi_{v}^{(j)}\right\|^{2},
\end{aligned}
$$

hence, replacing (59) into (58), we have:

$$
S_{v}^{(j)} \geq-\frac{1}{\rho b}\left|\psi_{v}^{(j)}\left(k_{v_{1}}-1\right) \psi_{v}^{(j)}\left(k_{v_{1}}\right)\right| .
$$

In so doing we have shown that each term $S_{v}^{(j)}$ satisfies the Popov inequality when $x(l) \in \Omega_{v}$. Notice that the same procedure can be used to show that (60) is still verified when $x(l) \notin \Omega_{v}$ simply by choosing $\Psi_{v}^{(j)}$ as

$$
\begin{gathered}
\Psi_{v}^{(j)}= \\
{\left[\begin{array}{cccccc}
\psi_{v}^{(j)}\left(k_{v_{1}}\right) & \psi_{v}^{(j)}\left(k_{v_{1}}+1\right) & \cdots & \psi_{v}^{(j)}\left(k_{v_{1}+1}-1\right) & \vdots \\
\psi_{v}^{(j)}\left(k_{v_{2}}\right) & \psi_{v}^{(j)}\left(k_{v_{2}}+1\right) & \cdots & \psi_{v}^{(j)}\left(k_{v_{2}+1}-1\right) & \vdots \\
\cdots & \cdots & \cdots & \cdots & \cdots \\
\psi_{v}^{(j)}\left(k_{v_{\left(\theta_{v}-1\right)}}\right) & \psi_{v}^{(j)}\left(k_{\left.v_{\left(\theta_{v}-1\right)}+1\right)}\right) & \cdots & \psi_{v}^{(j)}\left(k_{v_{\left(\theta_{v}-1\right)}+1}-1\right) & \vdots \\
\psi_{v}^{(j)}\left(k_{v_{\theta_{v}}}\right) & \psi_{v}^{(j)}\left(k_{v_{\theta_{v}}}+1\right) & \cdots & \psi_{v}^{(j)}\left(k_{v_{\theta_{v}}+1}-2\right) & \psi_{v}^{(j)}\left(k_{v_{\theta_{v}}+1}-1\right)
\end{array}\right]^{T} .}
\end{gathered}
$$

Since $S_{v}^{(j)}$ verifies the Popov inequality for all time instants, it follows that $S_{v}$ and $S$ in (41) satisfy the Popov inequality as well for some proper scalar constants.

Finally the same procedure can be used to prove that also $\widehat{S}$ verifies the Popov inequality. Indeed, this can be proved by replacing in the previous derivation $L_{v}^{(j)}$ with $\widehat{L}_{\widehat{v}}^{(j)}, \Omega_{v}$ with $\widehat{\Omega}_{\widehat{v}}$, the index $v$ with the index $\widehat{v}$ and $\delta_{v}^{(j)}$ with $\widehat{\delta}_{\widehat{v}}^{(j)} \triangleq \widehat{a}_{\widehat{v}}^{(j)}-\widehat{a}_{\widehat{0}}^{(j)}$.

\subsection{Asymptotic convergence of the closed loop error system}

We know from Subsection 4.3 that there exists $\gamma \in \mathbb{R}$ such that:

$$
\sum_{l=0}^{k} y_{e}(l+1) \xi(l+1) \leq \gamma^{2}, \quad \forall k \geq 0 .
$$


Hence, summing $y_{e}(0) \xi(0)$ to both sides, summing up to $k-1$ and using (21) and (25), one gets:

$$
\begin{aligned}
& \sum_{l=0}^{k} y_{e}(l) \xi(l) \leq \gamma^{2}+y_{e}(0) \xi(0)=\gamma^{2}+x_{e}^{T}(0) P x_{e}(0)-x_{e}(0)^{T} P \tilde{x}_{e}(0)= \\
& =\gamma^{2}+\left(x_{e}(0)-\frac{1}{2} \tilde{x}_{e}(0)\right)^{T} P\left(x_{e}(0)-\frac{1}{2} \tilde{x}_{e}(0)\right)-\frac{1}{4} \tilde{x}_{e}^{T}(0) P \tilde{x}_{e}(0) .
\end{aligned}
$$

Let us now consider (30). Summing up from 0 to $k$ results in:

$$
\begin{aligned}
\sum_{l=0}^{k} y_{e}(l) \xi(l)= & \frac{1}{2} \tilde{x}_{e}^{T}(k+1) P \tilde{x}_{e}(k+1)-\frac{1}{2} \tilde{x}_{e}^{T}(0) P \tilde{x}_{e}(0)+ \\
& +\frac{1}{2} \sum_{l=0}^{k} x_{e}^{T}(l) Q(k) x_{e}(l)+\frac{1}{2} p_{n n} \sum_{l=0}^{k} \xi^{2}(l) .
\end{aligned}
$$

Moreover, recalling (27), we define

$$
\lambda_{Q}:=\min _{\widehat{i}=0, \ldots, \widehat{M}-1}\left\{\lambda_{\widehat{i} 1}, \ldots, \lambda_{\widehat{i n}} ; \operatorname{det}\left(Q_{\widehat{i}}-\lambda \mathbb{I}\right)=0\right\},
$$

which is positive due to the fact that all the matrices $Q_{\widehat{i}}$ are positive definite by Assumption 1 . Then, taking again into account that $P$ is positive definite, it follows from (63) that:

$$
\sum_{l=0}^{k} y_{e}(l) \xi(l) \geq-\frac{1}{2} \tilde{x}_{e}^{T}(0) P \tilde{x}_{e}(0)+\frac{1}{2} \lambda_{Q} \sum_{l=0}^{k} x_{e}^{T}(l) x_{e}(l)+\frac{1}{2} p_{n n} \sum_{l=0}^{k} \xi^{2}(l) .
$$

Now, putting together (62) and (65), rearranging terms and taking limits for $k \rightarrow+\infty$ we obtain:

$$
\begin{aligned}
& \gamma^{2}+\left(x_{e}(0)-\frac{1}{2} \tilde{x}_{e}(0)\right)^{T} P\left(x_{e}(0)-\frac{1}{2} \tilde{x}_{e}(0)\right)+\frac{1}{4} \tilde{x}_{e}^{T}(0) P \tilde{x}_{e}(0) \geq \\
& \geq \lim _{k \rightarrow+\infty}\left[\frac{1}{2} \lambda_{Q} \sum_{l=0}^{k} x_{e}^{T}(l) x_{e}(l)+\frac{1}{2} p_{n n} \sum_{l=0}^{k} \xi^{2}(l)\right] .
\end{aligned}
$$

Furthermore, letting $\rho(P)$ stand for the spectral radius of $P$, one gets from (66) that

$$
\begin{aligned}
& \gamma^{2}+\left(\left\|x_{e}(0)-\frac{1}{2} \tilde{x}_{e}(0)\right\|^{2}+\frac{1}{4}\left\|\tilde{x}_{e}(0)\right\|^{2}\right) \rho(P) \geq \\
& \geq \lim _{k \rightarrow+\infty}\left[\frac{1}{2} \lambda_{Q} \sum_{l=0}^{k}\left\|x_{e}(l)\right\|^{2}+\frac{1}{2} p_{n n} \sum_{l=0}^{k} \xi^{2}(l)\right] .
\end{aligned}
$$

As both $\lambda_{Q}$ and $p_{n n}$ are positive, it follows immediately that:

$$
\lim _{k \rightarrow+\infty}\left\|x_{e}(k)\right\|=0, \quad \forall x_{e}(0), \tilde{x}_{e}(0),
$$

which proves the convergence of all error trajectories towards the origin from any initial condition. Additional results derived, respectively, from (66) and (21), also for any initial conditions, are:

$$
\lim _{k \rightarrow+\infty} \xi(k)=0, \quad \lim _{k \rightarrow+\infty} y_{e}(k)=0 .
$$




\section{FURTHER EXTENSIONS}

Assumption 1, aimed at ensuring the passivity of subsystem $\Sigma_{1}$ in the sense of equation (29), is equivalent to requiring Q-stability of the reference model. Hence, according to Remark 1, when the reference model is LTI this is equivalent to its dynamical matrix being Hurwitz. Nevertheless, for generic PWL reference models, Assumption 1 becomes a stronger requirement, namely, the existence of a common quadratic Lyapunov function for the unforced reference model system, which may yield the unfeasibility of the LMI problem (6).

Such a constraint can be relaxed taking advantage of the switching nature of the reference model. Hence, for a generic partition $\left\{\widehat{\Omega}_{\widehat{i}}\right\}_{\widehat{i} \in \widehat{\mathcal{M}}}$ of $\mathbb{R}^{n}$, we can seek for a PWQ Lyapunov function, while a further relaxation can be achieved when the partition is polyhedral. The latter is inspired on the approach introduced in [7], where sufficient LMI conditions for PWQ passivity of PWA systems are derived taking into account only allowed transitions between switching regions in one time-step (see also [18]). Thus, it is possible to state the following results.

Theorem 2

Assume that the reference model (3) is PWQ-stable [11], i.e. there exists a set of symmetric, positive definite matrices $P_{\widehat{i}}>0$, for all $\widehat{i} \in \widehat{\mathcal{M}}$, such that the following LMI is verified:

$$
\widehat{A}_{\hat{i}}^{T} P_{\widehat{j}} \widehat{A}_{\widehat{i}}-P_{\widehat{i}}<0, \quad \forall(\widehat{i}, \widehat{j}) \in \widehat{\mathcal{M}} \times \widehat{\mathcal{M}}
$$

Then, given system (1) and a reference model of the form (3), the adaptive law (7)-(14) with

$$
y_{e} \triangleq B_{e}^{T} P_{\widehat{i}} x_{e}, \quad x_{e} \triangleq \widehat{x}-x, \quad \text { when } \quad \widehat{x}(k) \in \widehat{\Omega}_{\widehat{i}},
$$

renders the origin of the closed-loop error dynamics globally asymptotically stable.

Proof

The proof follows that of Theorem 1, the main differences being:

- The common quadratic storage function (26) in Lemma 2 becomes a PWQ function [7] of the form

$$
V\left(\tilde{x}_{e}(k)\right)=\frac{1}{2} \tilde{x}_{e}^{T}(k) P(k) \tilde{x}_{e}(k), \quad \text { with } P(k)=P_{\widehat{i}} \text { when } \widehat{x}(k) \in \widehat{\Omega}_{\widehat{i}} .
$$

- Matrix $Q(k)$ defined in (27) becomes

$$
Q(k)=Q_{\widehat{i} \hat{j}}=P_{\widehat{i}}-\widehat{A}_{\hat{i}}^{T} P_{\widehat{j}} \widehat{A}_{\widehat{i}} \text { when } \widehat{x}(k) \in \widehat{\Omega}_{\widehat{i}}, \widehat{x}(k+1) \in \widehat{\Omega}_{\widehat{j}},
$$

and $\lambda_{Q}$ defined in (64) changes to:

$$
\lambda_{Q}:=\min _{\widehat{i}, \widehat{j}=0, \ldots, \widehat{M}-1}=\left\{\lambda_{\widehat{i} \hat{j}, 1}, \ldots, \lambda_{\widehat{i} \widehat{j}, n} ; \operatorname{det}\left(Q_{\widehat{i} \hat{j}}-\lambda \mathbb{I}\right)=0\right\},
$$

- The matrix element $p_{n n}$ in (30) becomes

$$
p_{n n}(k)=p_{\widehat{i}, n n} \quad \text { when } \widehat{x}(k) \in \widehat{\Omega}_{\widehat{i}},
$$

where $p_{\widehat{i}, n n}$ denotes the matrix element of $P_{\widehat{i}}$ located in the $(n, n)$ position. Correspondingly, $p_{n n}$ appearing in (66) is to be re-defined as:

$$
p_{n n}:=\min \left\{p_{\widehat{i}, n n}, \widehat{i} \in \widehat{\mathcal{M}}\right\},
$$

and it is positive due to the positive definiteness of the matrices $P_{\widehat{i}}$.

- Matrix $P$ in (66) is replaced by $P(0)$. 
- The spectral radius $\rho(P)$ in (67) is to be replaced by

$$
\tilde{\rho}:=\max _{\widehat{i} \in \widehat{\mathcal{M}}}\left\{\rho\left(P_{\widehat{i}}\right)\right\} .
$$

The fulfillment of the LMI (68) implicitly assumes that a state transition in one time step is possible between all regions of the partition, but this is not usually true. Then, if one is able to compute the subset $\mathcal{T}$ of ordered pairs $(\widehat{i}, \widehat{j})$, with $\widehat{i}, \widehat{j}=0,1, \ldots, \widehat{M}-1$, such that a transition from the region $\widehat{\Omega}_{\widehat{i}}$ at any time $k$ to $\widehat{\Omega}_{\widehat{j}}$ at time $k+1$ is allowed to occur along system trajectories, (68) is to be required only for $\mathcal{T}$. This is precisely the case with polyhedral partitions, which allows the computation of $\mathcal{T}$ via reachability analysis [7]. Hence, the following result is straightforward:

Corollary 1

Assume that $\left\{\widehat{\Omega}_{\widehat{i}}\right\}_{\widehat{i} \in \widehat{M}}$ is defined as:

$$
\widehat{\Omega}_{\widehat{i}}=\left\{\widehat{x} \in \mathbb{R}^{n}: \quad F_{\widehat{i}} \widehat{x} \geq f_{\widehat{i}}\right\}, \quad \widehat{i}=0,1, \ldots, \widehat{M}-1,
$$

$F_{\widehat{i}}, f_{\widehat{i}}$ being constant matrices and vectors, respectively, while the inequality is to be met componentwise. Then, let

$$
\mathcal{T}:=\left\{(\widehat{i}, \widehat{j}) \in \widehat{\mathcal{M}} \times \widehat{\mathcal{M}} ; \quad \exists \widehat{x} \in \mathbb{R}^{n}, r \in \mathbb{R} \text { such that } \widehat{x} \in \widehat{\Omega}_{\widehat{i}}, \quad \widehat{A}_{\hat{i}} \widehat{x}+\widehat{B} r \in \widehat{\Omega}_{\widehat{j}}\right\} .
$$

Assume now that the reference model (3) is $\mathcal{T}$-PWQ stable, i.e. there exists a set of symmetric positive definite matrices $P_{\widehat{i}}>0$, for all $\widehat{i} \in \widehat{\mathcal{M}}$, such that the following LMI is verified:

$$
\widehat{A}_{\hat{i}}^{T} P_{\widehat{j}} \widehat{A}_{\widehat{j}}-P_{\widehat{i}}<0, \quad \forall(\widehat{i}, \widehat{j}) \in \mathcal{T} .
$$

Then, the adaptive law (7)-(14) with

$$
y_{e} \triangleq B_{e}^{T} P_{\widehat{i}} x_{e}, \quad x_{e} \triangleq \widehat{x}-x, \quad \text { when } \quad \widehat{x}(k) \in \widehat{\Omega}_{\widehat{i}} .
$$

guarantees global asymptotic stability of the closed-loop error dynamics of (1)-(3).

\section{NUMERICAL VALIDATION}

The effectiveness of the proposed discrete-time MCS algorithm is shown in this Section through representative numerical examples. In particular, the approach will be tested here both in the case of a linear and a piecewise linear reference model.

The plant is a PWL system in canonical form:

$$
x(k+1)=\left\{\begin{array}{l}
{\left[\begin{array}{cc}
0 & 1 \\
-0.05 & 0.6
\end{array}\right] x(k)+\left[\begin{array}{l}
0 \\
1
\end{array}\right] u(k) \quad \text { if } \quad x(k) \in \Omega_{0},} \\
{\left[\begin{array}{cc}
0 & 1 \\
-0.12 & 0.8
\end{array}\right] x(k)+\left[\begin{array}{l}
0 \\
1
\end{array}\right] u(k) \text { if } \quad x(k) \in \Omega_{1},} \\
{\left[\begin{array}{cc}
0 & 1 \\
-0.175 & 0.95
\end{array}\right] x(k)+\left[\begin{array}{l}
0 \\
1
\end{array}\right] u(k) \text { if } \quad x(k) \in \Omega_{2},} \\
{\left[\begin{array}{cc}
0 & 1 \\
-0.32 & 1.2
\end{array}\right] x(k)+\left[\begin{array}{l}
0 \\
1
\end{array}\right] u(k) \quad \text { if } \quad x(k) \in \Omega_{3},}
\end{array}\right.
$$




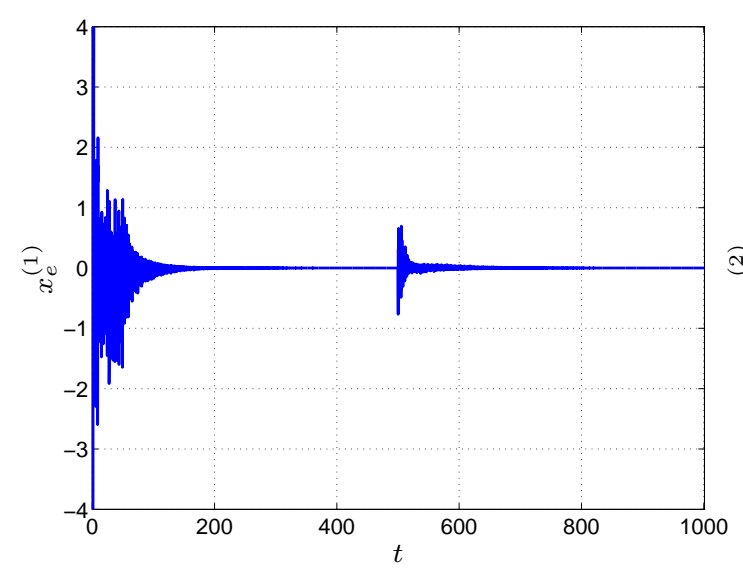

(a)

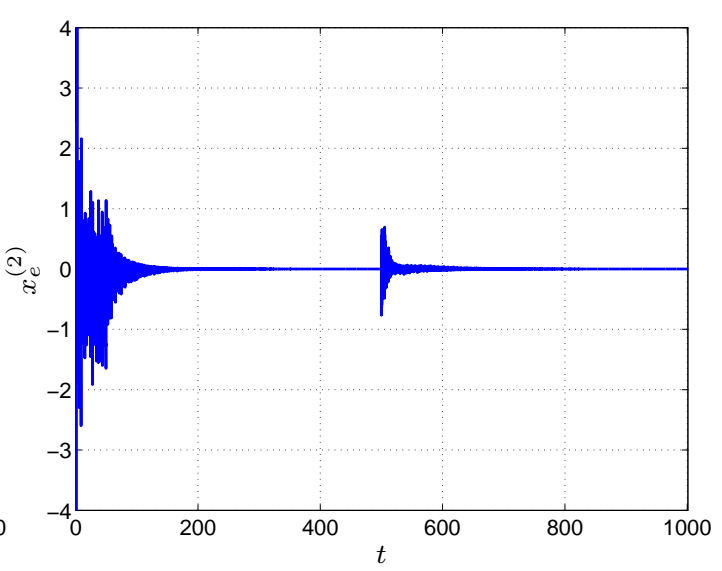

(b)

Figure 2. LTI ref. model. (a), (b): Time history of the tracking errors.

where

$$
\begin{aligned}
& \Omega_{0} \triangleq\left\{x: H_{0}^{T} x<0\right\} \cap\left\{x: H_{1}^{T} x \geq 0\right\}, \\
& \Omega_{1} \triangleq\left\{x: H_{0}^{T} x \geq 0\right\} \cap\left\{x: H_{1}^{T} x \geq 0\right\}, \\
& \Omega_{2} \triangleq\left\{x: H_{0}^{T} x \geq 0\right\} \cap\left\{x: H_{1}^{T} x<0\right\}, \\
& \Omega_{3} \triangleq\left\{x: H_{0}^{T} x<0\right\} \cap\left\{x: H_{1}^{T} x<0\right\},
\end{aligned}
$$

with $H_{0}^{T}=\left[\begin{array}{ll}-1 & 1\end{array}\right], H_{1}^{T}=\left[\begin{array}{ll}1 & 1\end{array}\right]$ and $x(0)=\left[\begin{array}{ll}2 & 1\end{array}\right]^{T}$.

Note that, as is typical with MRAC approaches, particular care must be taken when implementing the controller with the so-called one-delay problem [1]. The problem arises when computing the control gains at the current time instant $k$ according to equations (8)-(9), which depend on the value of $y_{e}$ at $k+1$. To solve this problem, Landau himself proposed as a practical solution to use instead a running estimate of $y_{e}(k+1)$ [1]. Here we follow precisely the same approach by setting:

$$
y_{e}(k+1) \approx \frac{y_{e}(k)}{1+p_{n n} b(\alpha+\beta) w^{\top}(k) w(k)} .
$$

Despite its simplicity, this choice yields a successful implementation of the control strategy. Concerning the estimation of the unknown parameter $b$, and again in accordance with Landau [1], $b$ can be replaced by a value that falls within its range of variation, which is assumed to be known.

Finally we remark that, as always happens when dealing with the MCS approach, the parameters of the controller are chosen heuristically as a trade off between convergence time and reactivity of the control actions. Our choice here is $\rho=100, \alpha=100$ and $\beta=10$, which yields the standard empiric ratio $\alpha / \beta=10$ suggested in [9].

\subsection{Case 1: LTI reference model}

As a first attempt for the validation of the adaptive switched control law, an LTI reference model is adopted. Hence, the target is to make the PWS plant behave as a smooth reference model of the form:

$$
\widehat{x}(k+1)=\left[\begin{array}{cc}
0 & 1 \\
-0.1 & 0.7
\end{array}\right] \widehat{x}(k)+\left[\begin{array}{c}
0 \\
1.2
\end{array}\right] r(k),
$$

the input signal $r$ being sinusoidal, namely $r=2 \sin (2 k)$, while the initial conditions are set to $\widehat{x}(0)=\left[\begin{array}{ll}0 & 0\end{array}\right]^{T}$. Assumption 1 is fulfilled, since the dynamic matrix of the reference model is Hurwitz. 


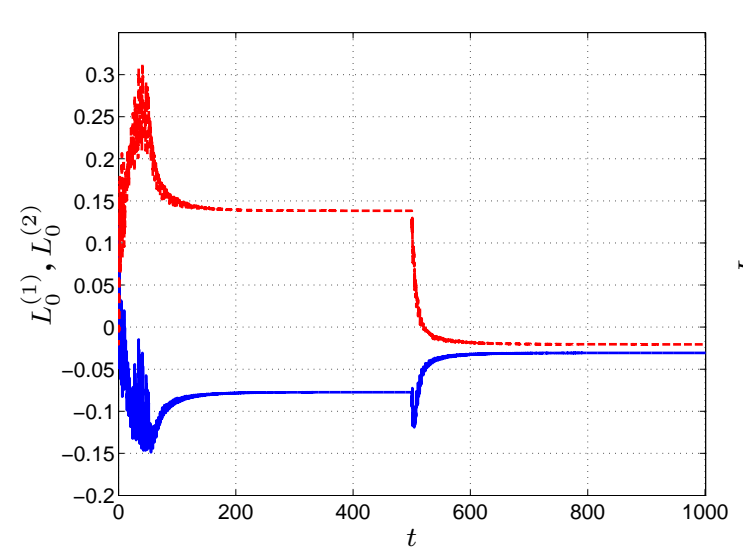

(a)

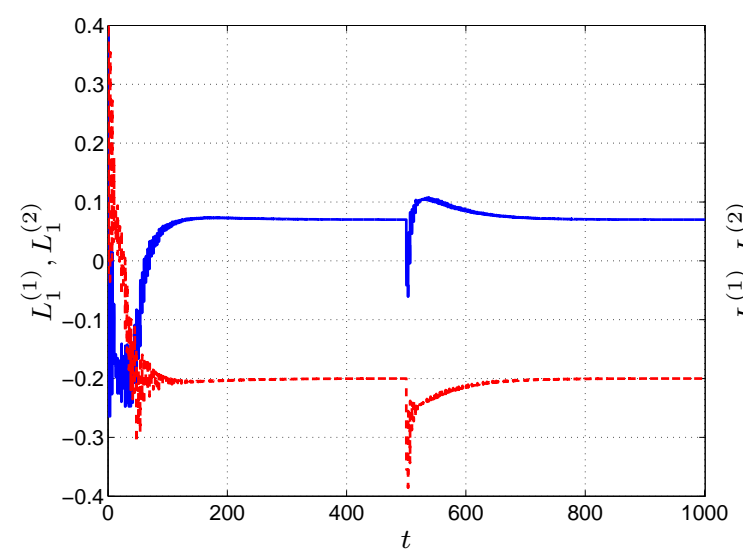

(c)

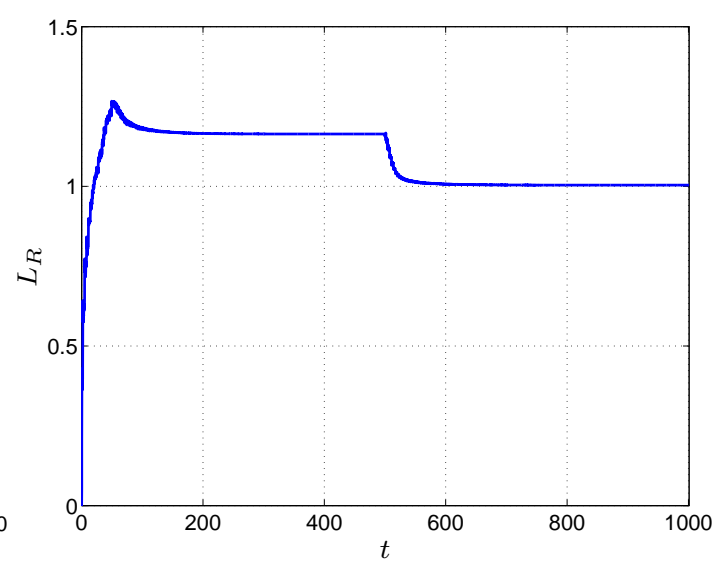

(b)

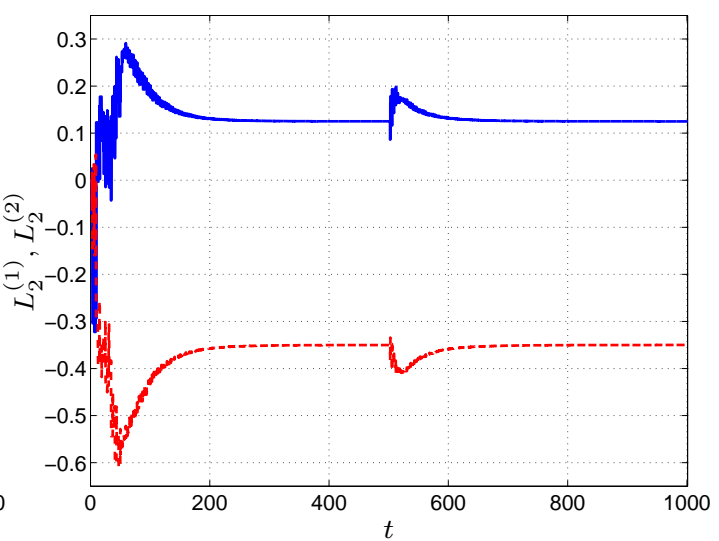

(d)

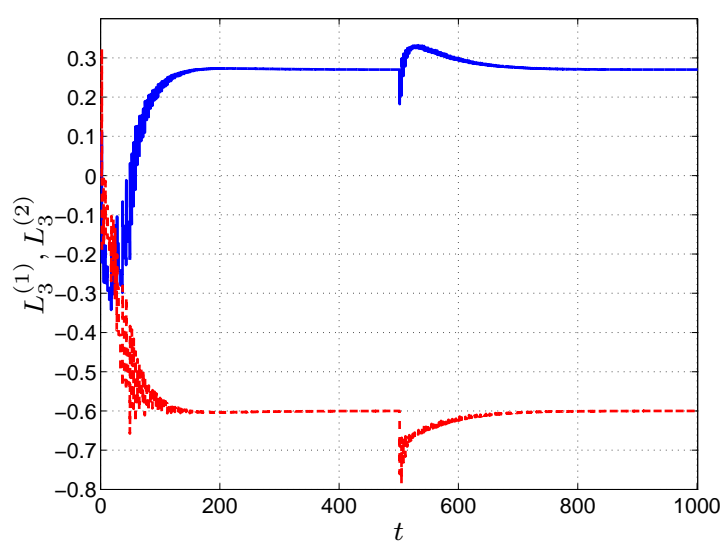

(e)

Figure 3. LTI ref. model. Adaptive gains related to plant modes. (a) $L_{0}: L_{0}^{(1)}$ (blue solid), $L_{0}^{(2)}$ (red dashed); (b) $L_{R}$; (c) $L_{1}: L_{1}^{(1)}$ (blue solid), $L_{1}^{(2)}$ (red dashed); (d) $L_{2}: L_{2}^{(1)}$ (blue solid), $L_{2}^{(2)}$ (red dashed); (e) $L_{3}: L_{3}^{(1)}$ (blue solid), $L_{3}^{(2)}$ (red dashed).

Figure 2 shows the tracking error evolution for both the state variables and their convergence to zero. Notice that, in order to numerically test the robustness of the control approach, the presence of a perturbation in the plant parameters has been also explicitly considered in the simulation 


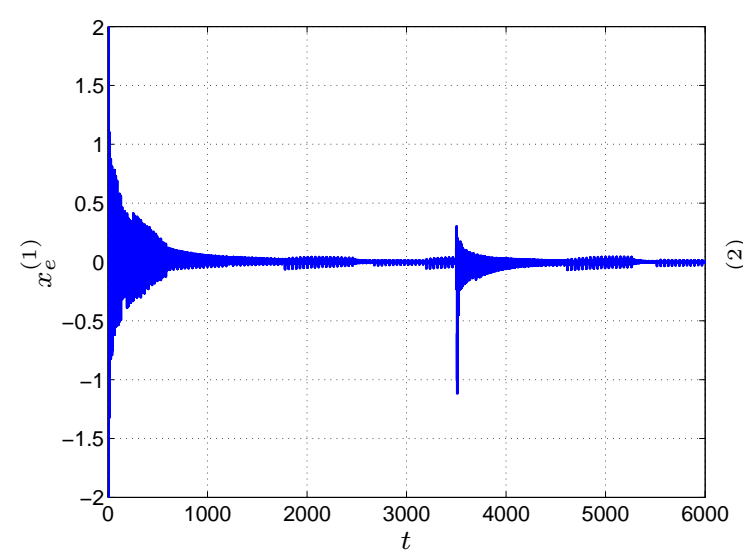

(a)

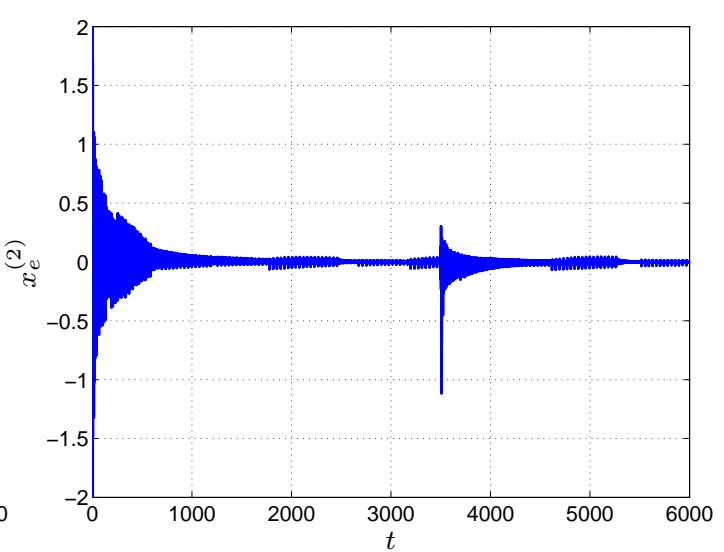

(b)

Figure 4. PWL ref. model. (a), (b) Time history of the tracking errors.

scenario. Specifically, the behavior in case of a sudden and simultaneous change of $20 \%$ in all plant parameters values, with respect to their nominal ones, occurring at 500 [s], is investigated. In Figure 2 it is evident that the control algorithm is able to reject this unexpected parameter variations and, through adaptation, to achieve again excellent tracking performance.

All the adaptive gains converge asymptotically to a finite value once the tracking error reduces to zero (see Figure 2) and, in the presence of a disturbance, converge to steady-state values after it has been rejected (see Figure 3). Notice also that the switching part of the controller, $L_{\Sigma}$, depends only on those switching terms associated with commutations of the plant, since the reference model is smooth.

\subsection{Case 2: PWL reference model}

The piecewise adaptive feedback law has been designed to deal with generic multimodal reference models characterized by a number of modes that can be different from the ones of the plant. To show the effectiveness of the control approach in this case, we select the following bimodal system as reference model:

$$
\widehat{x}(k+1)=\left\{\begin{array}{c}
{\left[\begin{array}{cc}
0 & 1 \\
-0.1 & 0.7
\end{array}\right] \widehat{x}(k)+\left[\begin{array}{c}
0 \\
1.2
\end{array}\right] r(k) \quad \text { if } \quad \widehat{x}(k) \in \widehat{\Omega}_{0},} \\
{\left[\begin{array}{cc}
0 & 1 \\
-0.008 & 0.24
\end{array}\right] \widehat{x}(k)+\left[\begin{array}{c}
0 \\
1.2
\end{array}\right] r(k) \text { if } \quad \widehat{x}(k) \in \widehat{\Omega}_{1},}
\end{array}\right.
$$

where

$$
\left.\begin{array}{l}
\widehat{\Omega}_{0} \triangleq\left\{\widehat{x}: \widehat{H}_{0}^{T} \widehat{x}>0\right. \\
\widehat{\Omega}_{1} \triangleq\left\{\widehat{x}: \widehat{H}_{0}^{T} \widehat{x} \leq 0\right.
\end{array}\right\},
$$

with $\widehat{H}^{T}=\left[\begin{array}{ll}0.5 & 1\end{array}\right], r(k)=2 \sin (0.5 k)$ and $\widehat{x}(0)=\left[\begin{array}{ll}2 & -1\end{array}\right]^{T}$. The fulfillment of Assumption 1 can be verified solving off-line the LMI problem (6) by means of MATLAB's LMI toolbox, which yields

$$
P=10^{8}\left(\begin{array}{cc}
2.8860 & -0.2580 \\
-0.2580 & 7.5618
\end{array}\right)
$$

The new adaptive controller guarantees, through adaptation, excellent tracking performance of the state variables of the reference model as confirmed in Figure 4 that shows the error tracking evolution for both the state variables. 


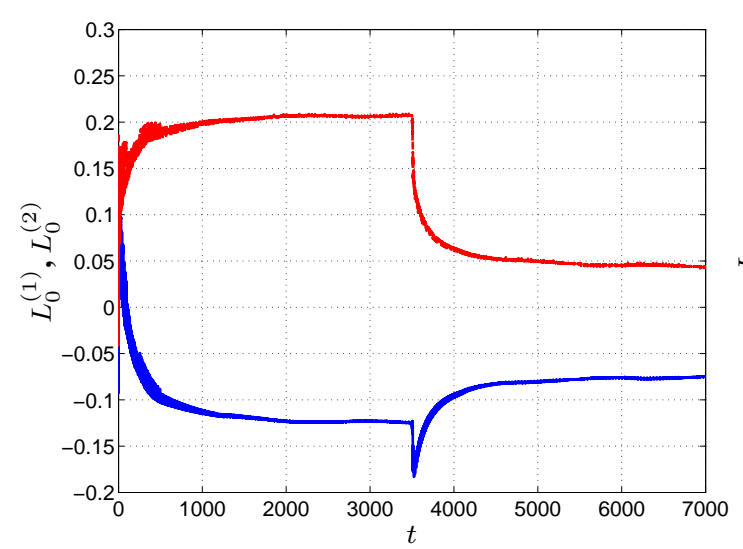

(a)

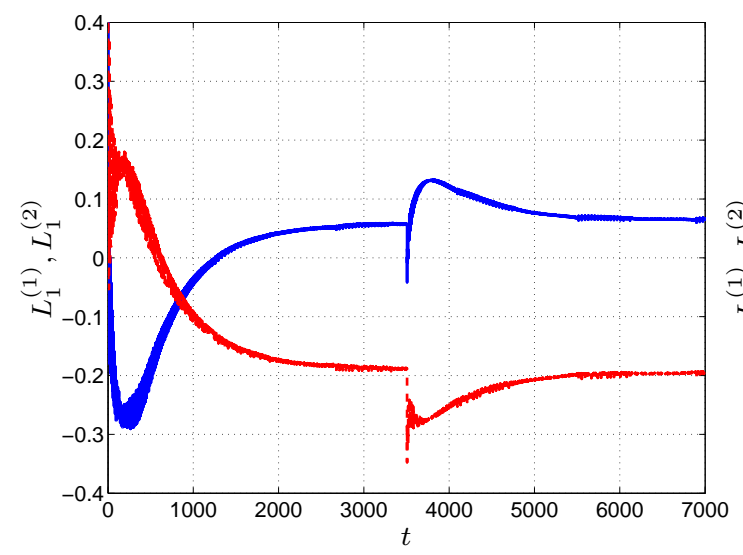

(c)

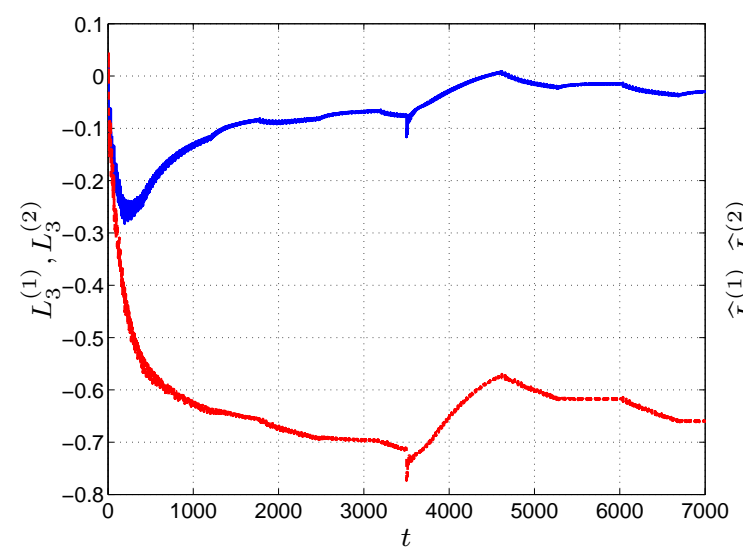

(e)

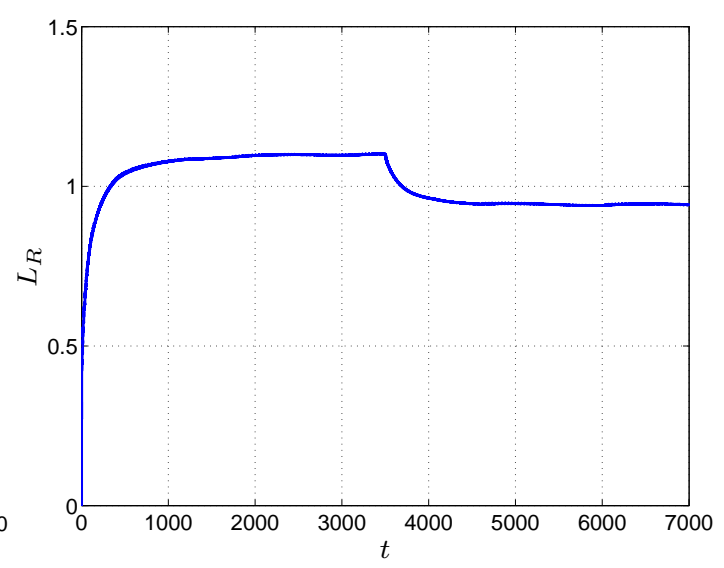

(b)

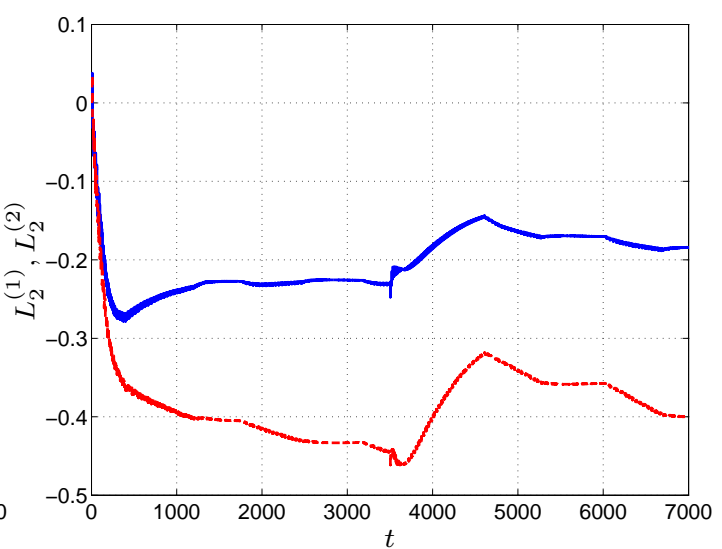

(d)

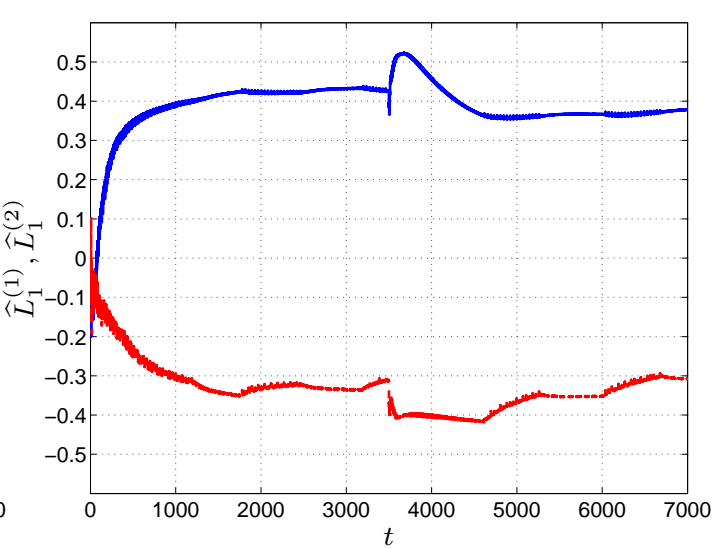

(f)

Figure 5. PWL ref. model. Adaptive gains related to plant modes. (a) $L_{0}: L_{0}^{(1)}$ (blue solid), $L_{0}^{(2)}$ (red dashed); (b) $L_{R}$; (c) $L_{1}: L_{1}^{(1)}$ (blue solid), $L_{1}^{(2)}$ (red dashed); (d) $L_{2}: L_{2}^{(1)}$ (blue solid), $L_{2}^{(2)}$ (red dashed); (e) $L_{3}: L_{3}^{(1)}$ (blue solid), $L_{3}^{(2)}$ (red dashed); (f) $\widehat{L}_{1}: \widehat{L}_{1}^{(1)}$ (blue solid), $\widehat{L}_{1}^{(2)}$ (red dashed).

As in the previous case of study, the effect of a sudden and simultaneous change of $20 \%$ in the parameters values is also investigated in the numerical analysis. As shown in Figure 4 the control 
algorithm is robust with respect to these abrupt parameter variations occurring at 3500 [s]. The presence of a negligible residual relative error is due to the use of the simple approach proposed by Landau in [1] to solve the one-delay problem, as discussed above.

All adaptive gains, both switching and continuous, remain bounded (see Figure 5). Note that the gains converge again to a finite steady state value, with the disturbance being effectively rejected.

\section{CONCLUSIONS}

We have addressed an existing gap in the theory of MRAC approaches by proposing a switched MRAC scheme to control discrete-time PWL plants. The idea is to consider a control law composed of three different adaptive action. Namely, a smooth adaptive feedforward term and a smooth adaptive feedback term are complemented by a switched adaptive actions. Using the theory of passivity, we have shown that, under appropriate conditions, such a controller can guarantee global asymptotic stability of the error system. The proposed controller was tested on two representative examples showing excellent performance not only when the reference model is LTI but also when it is itself a PWL system.

We envisage that our controller could be practically used in all of those applications where, for example, there is the need to make a PWL plant behave as a smooth system. In that case, it suffices to choose an LTI reference model and implement the MRAC controller proposed in this paper to guarantee good performance and robustness to unwanted parameter variations, noise and unmodelled dynamics.

\section{ACKNOWLEDGEMENT}

J.M. Olm is partially supported by the spanish Ministerio de Ciencia e Innovación under project DPI201015110 .

\section{REFERENCES}

1. Landau ID. Adaptive Control. The Model Reference Approach. Marcel Dekker, 1979.

2. di Bernardo M, Budd CJ, Champneys AR, Kowalczyk P. Piecewise-Smooth Dynamical Systems: Theory and Applications. Springer-Verlag, 2007.

3. di Bernardo M, Montanaro U, Santini S. Novel switched model reference adaptive control for continuous piecewise affine systems. 47th IEEE Conference on Decision and Control, 2008; 1925-1930.

4. Sang Q, Tao G. Adaptive control of piecewise linear systems: the state tracking case. American Control Conference, 2010; 4040-4045.

5. di Bernardo M, Montanaro U, Santini S. Minimal control synthesis adaptive control of continuous bimodal piecewise affine systems. SIAM Journal on Control and Optimization 2010; 48(7):4242-4261.

6. Zhao J, Hill DJ. Dissipativity theory for switched systems. IEEE Transactions on Automatic Control 2008; 53(4):941-953.

7. Bemporad A, Bianchini G, Brogi F. Passivity analysis and passification of discrete-time hybrid systems. IEEE Transactions on Automatic Control 2008; 53(4):1004-1009.

8. di Bernardo M, Montanaro U, Santini S. Novel hybrid MRAC-LQ control schemes: synthesis, analysis and applications. International Journal of Control 2008; 81(6):940-961.

9. Stoten DP, Benchoubane H. Robustness of minimal controller synthesis algorithm. International Journal of Control 1990; 51(4):851-861.

10. di Bernardo M, di Gennaro F, Olm JM, Santini S. Discrete-time minimal control synthesis adaptive algorithm. International Journal of Control 2010; 83(4):2641-2657.

11. Mignone D, Ferrari-Trecate G, Morari M. Stability and stabilization of piecewise affine and hybrid systems: An LMI approach. 39th IEEE Conference on Decision and Control, 2000; 504-509.

12. Landau ID, Lozano R, M’Saad M. Adaptive Control. Springer-Verlag, London, 1998.

13. Tao G, Lewis FF. Adaptive Control of Nonsmooth Dynamic Systems. Springer, London, 2001.

14. Liberzon D. Switching in Systems and Control. Birkhäuser, 2003.

15. Brogliato B. Nonsmooth Mechanics: Models, Dynamics and Control. Springer-Verlag, 1999.

16. van der Schaft AJ, Schumacher H. An Introduction to Hybrid Dynamical Systems. Springer, 2000.

17. Branicky MS. Multiple Lyapunov functions and other analysis tools for switched and hybrid systems. IEEE Transactions on Automatic Control 1998; 43(4):475-482.

18. Lee JW, Dullerud GE. Uniform stabilization of discrete-time switched and Markovian jump linear systems. Automatica 2006; 42(2):205-218. 
19. Byrnes CI, Lin W. Losslessness, feedback equivalence, and the global stabilization of discrete-time nonlinear systems. IEEE Transactions on Automatic Control 1994; 39(1):83-98.

\section{A. BASICS OF DISCRETE-TIME PASSIVITY}

The material in this section has been mainly extracted from [19] and [12]. Consider a generic state-space system $\Sigma$ of the form:

$$
\Sigma:=\left\{\begin{array}{ccc}
x(k+1) & = & f(x(k), u(k)) \\
y(k) & = & h(x(k), u(k))
\end{array}\right.
$$

Definition 1

$\Sigma$ is passive if there exists a nonnegative function $V: \mathbb{R}^{n} \longrightarrow \mathbb{R}$, with $V(0)=0$, such that the following dissipation inequality holds along any of its possible trajectories:

$$
V(x(k+1))-V(x(k)) \leq y^{T}(k) u(k), \quad \forall u(k), \forall k \geq 0 .
$$

$V$ is known as a storage function for $\Sigma$.

Notice that (75) holds if and only if

$$
V(x(k+1))-V(x(0)) \leq \sum_{i=0}^{k} y^{T}(i) u(i), \quad \forall u(k), \forall x(0) \text { and } \forall k \geq 0 .
$$

Let us now define subclasses of passive systems where the inequality may be strict:

Definition 2

Let $\Sigma$ be a passive system with storage function $V$. Assume that there exist $\delta_{1}, \delta_{2} \geq 0$ such that, $\forall u(k)$ and $\forall k \geq 0$

$$
V(x(k+1))-V(x(k)) \leq y^{T}(k) u(k)-\delta_{1} x^{T}(k) x(k)-\delta_{2} u^{T}(k) u(k)
$$

- If $\delta_{1}>0$, then $\Sigma$ is state strictly passive.

- If $\delta_{2}>0$, then $\Sigma$ is input strictly passive.

When $\Sigma$ is an input-output system, the definition of passivity is reformulated from (76) as follows:

\section{Definition 3}

$\Sigma$ is passive if there exists $\gamma \in \mathbb{R}$ such that

$$
\sum_{i=0}^{k} y^{T}(i) u(i) \geq-\gamma^{2}, \quad \forall k \geq 0
$$

Input strict passivity can be equivalently stated.

Consider now the discrete linear time-varying system

$$
\begin{aligned}
x(k+1) & =A(k) x(k)+B u(k) \\
y(k) & =C(k) x(k)+D(k) u(k) .
\end{aligned}
$$

Lemma 3

[12] System (77)-(78) is passive if one of the following equivalent conditions hold:

i) There exist time-varying matrices $P(k), Q(k), R(k), S(k)$, with $P(k)$ positive definite and $Q(k), R(k)$ positive semidefinite, such that:

$$
\begin{aligned}
& A^{T}(k) P(k+1) A(k)-P(k)=-Q(k), \\
& C(k)-B^{T}(k) P(k+1) A(k)=S^{T}(k), \\
& D(k)+D^{T}(k)-B^{T}(k) P(k+1) B(k)=R(k), \\
& M(k)=\left(\begin{array}{cc}
Q(k) & S(k) \\
S^{T}(k) & R(k)
\end{array}\right) \geq 0,
\end{aligned}
$$

with $P(0)$ bounded. 
ii) The following equality is satisfied, $\forall k \geq 0$ :

$$
\begin{aligned}
y^{T}(k) u(k)= & \frac{1}{2} x^{T}(k+1) P(k+1) x(k+1)-\frac{1}{2} x^{T}(k) P(k) x(k)+ \\
& +\frac{1}{2}\left[\begin{array}{ll}
x^{T}(k) & u^{T}(k)
\end{array}\right] M(k)\left[\begin{array}{c}
x(k) \\
u(k)
\end{array}\right] .
\end{aligned}
$$

It is immediate from Lemma 3 that (77)-(78) is passive if it admits a quadratic storage function of the form

$$
V(x(k))=\frac{1}{2} x^{T}(k) P(k) x(k) .
$$

\section{B. MATHEMATICAL DERIVATION OF THE ERROR DYNAMICS}

In order to obtain the error dynamics equation (16), let us first rewrite the plant model and the reference model introduced in eqs. (1) and (3) as:

$$
\begin{aligned}
& x(k+1)=A(k) x(k)+B u(k), \\
& \widehat{x}(k+1)=\widehat{A}(k) \widehat{x}(k)+\widehat{B} r(k),
\end{aligned}
$$

where

$$
\begin{aligned}
& A(k)=A(x(k))=A_{i} \quad \text { if } \quad x(k) \in \Omega_{i}, \\
& \widehat{A}(k)=\widehat{A}(\widehat{x}(k))=\widehat{A}_{\widehat{i}} \quad \text { if } \quad \widehat{x}(k) \in \widehat{\Omega}_{\widehat{i}} .
\end{aligned}
$$

The tracking error dynamics are given by:

$$
\begin{aligned}
x_{e}(k+1) & =\widehat{x}(k+1)-x(k+1)= \\
& =\widehat{A}(k) x_{e}(k)+\widehat{B} r(k)+(\widehat{A}(k)-A(k)) x(k)-B u(k) .
\end{aligned}
$$

From (86), matrix $A(k)$ can be written as

$$
A(k)=A_{0}-\sum_{v=1}^{M-1} \sigma_{v}(k) \Delta A_{v} \quad, \Delta A_{v}=A_{0}-A_{v}, \quad v=1, \ldots, M-1,
$$

where functions $\sigma_{v}$ are defined in (5). Notice that if $x(k) \in \Omega_{i}$, then $\sigma_{i}(k)=1$ and $\sigma_{v}(k)=0$ when $v \neq i$; hence,

$$
A(k)=A_{0}-\Delta A_{i}=A_{0}-\left(A_{0}-A_{i}\right)=A_{i},
$$

as defined in (86). Analogously, $\widehat{A}(k)$ can be rewritten as:

$$
\widehat{A}(k)=\widehat{A}_{0}+\sum_{\widehat{v}=1}^{\widehat{M}-1} \widehat{\sigma}_{\widehat{v}}(k) \Delta \widehat{A}_{\widehat{v}} \quad, \Delta \widehat{A}_{\widehat{v}}=\widehat{A}_{\widehat{v}}-\widehat{A}_{0}, \quad \widehat{v}=1 \ldots, \widehat{M}-1,
$$

with $\widehat{\sigma}_{\widehat{v}}$ also defined in (5) in an analogous way as $\sigma_{v}$.

Replacing (89), (90) in (88) and rearranging terms we have:

$$
\begin{aligned}
x_{e}(k+1)= & \widehat{A}(k) x_{e}(k)+\left(\widehat{A}_{0}-A_{0}\right) x(k)+\left(\sum_{v=1}^{M-1} \sigma_{v}(k) \Delta A_{v}\right) x(k)+ \\
& +\left(\sum_{\widehat{v}=1}^{\widehat{M}-1} \widehat{\sigma}_{\widehat{v}}(k) \Delta \widehat{A}_{\widehat{v}}\right) x(k)+\widehat{B} r(k)-B u(k) .
\end{aligned}
$$

Now, since $A_{i}$ and $\widehat{A}_{\widehat{i}}$ are assumed to be in control canonical form (recall (2) and (4), respectively), the first $(n-1)$ rows of these matrices are identical. Hence:

$$
\widehat{A}_{0}-A_{0}=\left[\begin{array}{ccccc}
0 & 0 & \cdots & 0 & 0 \\
0 & 0 & \cdots & \vdots & 0 \\
\vdots & \vdots & \ddots & \vdots & \vdots \\
0 & 0 & \cdots & 0 & 0 \\
\delta_{0}^{(1)} & \delta_{0}^{(2)} & \cdots & \delta_{0}^{(n-1)} & \delta_{0}^{(n)}
\end{array}\right], \quad \delta_{0}^{(j)}=\widehat{a}_{0}^{(j)}-a_{0}^{(j)}, \quad j=1, \ldots, n
$$


On the other hand, recalling that $B_{e}^{T}=(0, \ldots, 0,1)$, it is immediate that:

$$
\widehat{A}_{0}-A=B_{e} B_{e}^{T}\left(\widehat{A}_{0}-A\right) .
$$

Moreover, as the matrices $\Delta A_{v}$ and $\widehat{\Delta} A_{\widehat{v}}$ introduced in (89) and (90), respectively, exhibit the structure (92) because of the canonical form assumption, we also have that:

$$
\begin{array}{ll}
\Delta A_{v}=B_{e} B_{e}^{T} \Delta A_{v}, & v=1, \ldots, M-1, \\
\widehat{\Delta} A_{\widehat{v}}=B_{e} B_{e}^{T} \widehat{\Delta} A_{\widehat{v}}, & \widehat{v}=1, \ldots, \widehat{M}-1 .
\end{array}
$$

Substituting (93)-(95) in (91) and noticing that $B=b B_{e}$ and $\widehat{B}=\widehat{b} B_{e}$ we get:

$$
\begin{aligned}
x_{e}(k+1)= & \widehat{A}(k) x_{e}(k)+B_{e} B_{e}^{T}\left(\widehat{A}_{0}-A_{0}\right) x(k)+\widehat{b} B_{e} r(k)-b B_{e} u(k)+ \\
& +\left(\sum_{v=1}^{M-1} \sigma_{v}(k) B_{e} B_{e}^{T} \Delta A_{v}\right) x(k)+\left(\sum_{\widehat{v}=1}^{\widehat{M}-1} \widehat{\sigma}_{\widehat{v}}(k) B_{e} B_{e}^{T} \Delta \widehat{A}_{\widehat{v}}\right) x(k) .
\end{aligned}
$$

The control strategy defined in (7)-(15) yields:

$$
\begin{aligned}
u(k) & =L_{F B}(k) x(k)+L_{R}(k) r(k)= \\
& =\left[L_{0}(k)+L_{\Sigma}(k)+\widehat{L}_{\widehat{\Sigma}}(k)\right] x(k)+L_{R}(k) r(k)= \\
& =\left[L_{0}(k)+\sum_{v=1}^{M-1} \sigma_{v}(k) L_{v}(k)+\sum_{\widehat{v}=1}^{\widehat{M}-1} \widehat{\sigma}_{\widehat{v}}(k) \widehat{L}_{\widehat{v}}(k)\right] x(k)+L_{R}(k) r(k)
\end{aligned}
$$

Replacing $u(k)$ in the former expression for $x_{e}(k)$ and rearranging terms we have that:

$$
\begin{aligned}
x_{e}(k+1)= & \widehat{A}(k) x_{e}(k)+B_{e}\left[B_{e}^{T}\left(\widehat{A}_{0}-A_{0}\right)-b L_{0}(k)\right] x(k)+ \\
& +B_{e}\left[\widehat{b}-b L_{R}(k)\right] r(k)+ \\
& +B_{e}\left[\sum_{v=1}^{M-1} \sigma_{v}(k)\left(B_{e}^{T} \Delta A_{v}-b L_{v}(k)\right) x(k)\right]+ \\
& +B_{e}\left[\sum_{\widehat{v}=1}^{\widehat{M}-1} \widehat{\sigma}_{\widehat{v}}(k)\left(B_{e}^{T} \Delta \widehat{A}_{\widehat{v}}-b \widehat{L}_{\widehat{v}}(k)\right) x(k)\right] .
\end{aligned}
$$

Finally, the closed-loop tracking dynamics (16) follows easily after introducing in (96) the functions defined in (18)-(20). 